\title{
Prolotherapy: A Non-Invasive Approach to Lesions of the Glenoid Labrum; A Non-Controlled Questionnaire Based Study
}

\author{
Ross A. Hauser ${ }^{*}$ Erin Dolan and Amos Orlofsky
}

Caring Medical and Rehabilitation Services, 715 Lake Street, Suite 600, Oak Park, IL 60301, USA

\begin{abstract}
Lesions of the glenoid labrum are a common cause of shoulder instability and a frequent finding in patients with shoulder pain. Management of these patients typically involves an attempt to avoid surgery through conservative treatment. However, there is currently a dearth of conservative options that promote labral healing. Regenerative injection therapies, including prolotherapy, have shown promise in the treatment of several musculoskeletal disorders, but have not previously been applied to glenoid labral tear. Here we review several important aspects of these lesions and present an initial case series of 33 patients with labral tear that were treated in our clinic with intra-articular injections of hypertonic dextrose. Patient-reported assessments were collected by questionnaire at a mean follow-up time of 16 months. Treated patients reported highly significant improvements with respect to pain, stiffness, range of motion, crunching, exercise and need for medication. All 31 patients who reported pain at baseline experienced pain relief, and all 31 who reported exercise impairment at baseline reported improved exercise capability. Patients reported complete relief of $69 \%$ of recorded symptoms. One patient reported worsening of some symptoms. Prolotherapy for glenoid labral tear appears to be a safe procedure that merits further investigation.
\end{abstract}

Keywords: Prolotherapy, glenoid labrum lesions, shoulder, hypertonic dextrose, musculosketetal repair, case report, regenerative injection therapy, glenoid labral tear.

\section{INTRODUCTION}

Shoulder pain is among the most common complaints of patients seeking musculoskeletal consultation in primary care. The shoulder is involved in $8-13 \%$ of athletic injuries [1], and in 2006 almost 7.5 million visits were made to physicians' offices due to shoulder pain [2]. Among the many pathologies that can give rise to shoulder pain, labral lesions are among the most prevalent findings. In a prospective study of 208 consecutive cases of shoulder pain in primary care, magnetic resonance arthrograms were performed on 93 patients, of whom $44(47 \%)$ were found to have glenoid labral tear [3]. In a retrospective study of 544 arthroscopies, labral lesions were diagnosed in $44 \%$ of cases [4]. In the majority of cases, labral lesions are found in association with other intra-articular pathologies, including rotator cuff tears, instability, impingement syndrome, HillSachs lesions, osteoarthritis and paralabral cysts [3-5]. Since definitive diagnosis of many of these conditions is not possible preoperatively, management of these patients is a challenge for the primary care provider.

Management of patients with glenohumeral joint disorders generally includes a recommended period of conservative treatment in an attempt to avoid arthroscopic surgery. There is little data available, however, with respect to the efficacy of conservative treatment, and guidelines for the design of non-operative treatment plans have not been

*Address correspondence to this author at the Caring Medical and Rehabilitation Services, 715 Lake Street, Suite 600, Oak Park, IL 60301, USA; Tel: 708-848-7789; Fax: 708-848-7763;

E-mail: JonesM@caringmedical.com developed. Also unknown is the number of patients who choose to live with symptoms rather than undergo surgery. While arthroscopic treatment of glenoid labral tears produces good to excellent outcomes in most patients [6], the likelihood of success can vary with patient characteristics, including age and physical activity [6-8]. These limitations, combined with the expense and potential complications of arthroscopic therapy $[9,10]$, suggest a need for further investigation of non-operative treatment modalities.

Both conservative and operative treatments of glenoid labral lesions are founded on the concept of labral healing to the glenoid. Recent studies in an animal model support the concept of labral healing during conservative treatment [11]. One approach to the augmentation of healing has involved the development of several procedures, collectively termed regenerative injection therapies, that are designed to promote intra-articular healing via the introduction of growth factors and other stimulants of inflammatory and healing processes to the intra-articular milieu. Here we review relevant aspects of glenoid labral tear and describe initial observations in a group of patients treated with regenerative injection (prolotherapy) for labral tears.

\section{GLENOID LABRUM: ANATOMY AND FUNCTION}

The glenoid labrum is a ring of fibrocartilaginous tissue that runs along the rim of the glenoid fossa. Inferiorly, the labrum is continuous with the articular cartilage of the glenoid, but superiorly may adopt a variety of morphologies, in which attachment to the articular cartilage is either firm, loose or absent $[4,12,13]$. This anatomic variation can render the diagnosis of superior labral tears problematic. Inappropriate "repair" of anatomic variants misdiagnosed as 
lesions has led to substantial stiffness, pain and loss of external rotation [14]. The labrum serves as an insertion point for the biceps tendon and the glenohumeral ligament. The biceps origin is also subject to anatomic variation [15].

The labrum has multiple functions that contribute to the stabilization of the glenohumeral joint. The bony structure of the joint is designed to optimize range of motion at the expense of stability, as the humeral head is four times larger than the glenoid cavity [16]. The labrum increases glenoid coverage, augmenting the articular surface area in contact with the humeral head by one third [17]. The resulting gain in glenoid concavity contributes to stabilization [18]. Anchoring of the biceps, triceps and capsuloligaments by the labrum also contributes to stability [18]. Finally, the labrum acts as a seal, resisting dislocation by the maintenance of negative intra-articular pressure [19]. In view of these multiple roles, the restoration and maintenance of the structural integrity of the labrum should be a central goal of treatment strategies for patients with labral tear.

The vascularity of the labrum is potentially a limiting factor in the healing capacity of this tissue. A recent scanning electron micrographic study revealed that, while the dense core of the labrum is avascular, this is surrounded by a looser peri-core zone that is often vascularized, particularly in the superoanterior labrum [20]. The authors supported this finding with a confocal study that identified corresponding concentrations of vimentin-positive cells. In contrast, earlier cadaver studies observed labral vascularity to be greatest in the inferior and posterior regions [21]. The labral vasculature is more prominent in the labral periphery than in the central region [17], and is more extensive in younger individuals [22]. Therefore, while further studies are required to clearly define the distribution of vascular supply within the labrum, findings to date indicate a potential for vascular support of the healing of labral lesions.

\section{LESIONS OF THE GLENOID LABRUM}

The majority of glenoid labral lesions are tears resulting from acute injury $[23,24]$. Humeral translation, for example during a fall on an outstretched arm, or motions that generate traction on the biceps and capsular insertions can all result in avulsive tears of the superior labrum. Forceful abduction, extension and external rotation of the shoulder can avulse the anterior labrum. Less commonly, posterior labral tears may result, for example, from axial loading of adducted, internally rotated arms. In 1923, Bankart described an "essential" lesion involving detachment of the labrum and capsule in the anteroinferior aspect of the joint [25]. Tears may also result from chronic repetitive overload, as occurs during participation in overhead sports. In addition to tears, degenerative lesions (fraying of the labrum) are observed in conjunction with degenerative joint disease. Degenerative lesions are associated with aging and are attributed to chronic repetitive compression overload [23].

Morphologically, tears may be classified as flap tears, incomplete split tears, bucket-handle tears, and "superior labrum anterior and posterior" (SLAP) tears, in which the injury begins in the posterior region and continues superiorly and anteriorly [24]. SLAP lesions are uncommon, occurring in 6 percent of arthroscopies [26], but are a frequent and disabling injury in high-performance overhead athletes and have consequently been a focus of recent studies in the diagnosis and treatment of glenoid labral tears. The etiology of these injuries is not well understood and can involve either excessive compression or traction [26]. The most common SLAP lesions are type II, in which the superior labrum and biceps tendon are each detached from the glenoid. The spectrum of SLAP lesions illustrates the difficulties in management of patients with labral tear. Type I SLAP tears, for example, constitute approximately 20 percent of SLAP lesions [26]. These tears, which are highly associated with rotator cuff lesions [4], are considered of low clinical relevance [27] and surgery for these patients may not be indicated [28]. However, type I SLAP tears are difficult to distinguish from type III and type IV preoperatively [28]. Consequently, inaccurate diagnosis can result in unnecessary arthroscopy.

While labral tears can occur in isolation, the majority of tears are found in the context of additional glenohumeral pathology [4,26]. Snyder et al. reported that 81 percent of patients with SLAP tears had other associated shoulder lesions, including impingement (47 percent) and rotator cuff lesions (39 percent) [26]. Kim et al. found that 88 percent of SLAP tears had associated pathologies, including rotator cuff tears, instability, Bankart lesions and osteoarthritis [4]. The complexity of the presentation of glenoid labral lesions presents a challenge to the design of appropriate, individualized surgical treatment in patients that elect arthroscopy, underscoring the potential value of improvements in conservative care.

\section{DIAGNOSIS}

The diagnosis of glenoid labral tears begins with history and physical examination. Patient history may indicate relevant acute events or sources of chronic stress. Patient reports of popping, clicking or catching can contribute to a diagnosis of labral tear [29]. A large number of physical examinations have been studied as tools for diagnosis, including the crank test, the active compression (O'Brien) test, the anterior slide test, the anterior apprehension test, Speed's test, Jobe's relocation test, and testing for tenderness in the bicipital groove. In a prospective study of 55 patients scheduled for shoulder surgery, Walsworth et al. found that various combinations of the crank test, active compression test, anterior slide test, and popping/catching/clicking were more useful than individual tests [29]. The specificity of these combinations ranged from 82 to 91 percent.

Magnetic resonance imaging (MRI) has emerged as the preferred method for the preoperative diagnosis of glenoid labral tears. Supplementation of this method with an intraarticular contrast agent (MRA) is frequently employed to provide greater accuracy, as assessed using arthroscopic diagnosis as a gold standard. In a recent meta-analysis of 60 studies, Smith found that the sensitivity and specificity of MRI for overall glenoid labral lesions were 76 percent and 87 percent, respectively, while for MRA the corresponding values were 88 percent and 93 percent [30]. The authors concluded that MRA provides a marginal improvement in diagnostic accuracy, at a cost of increased expense, invasiveness, and exposure to radiation.

Despite these advances, the complexity of labral tear presentation creates significant challenges for the use of imaging to avoid unnecessary arthroscopy. In particular, the 
accuracy of imaging is not equivalent for all of the common subtypes of labral lesions. In their meta-analysis, Smith found that the specificities of MRI for superior labral tear and for Bankart lesions were only 74 and 56 percent respectively. For MRA, these values increased to 75 and 80 percent [30]. As already discussed, the differentiation between types I, III, and IV SLAP tears can also be problematic, with important consequences for the planning of surgery [28].

\section{TREATMENT}

While there are no published guidelines for the treatment of glenoid labral lesions, most authors recommend an initial period of conservative treatment, including the use of analgesics, NSAIDs, rest, and physical therapy that incorporates an individualized exercise program for capsular stretching and muscular strengthening. There are, however, very few published findings with respect to the prevalence, duration, composition or efficacy of conservative treatment, and consequently there has been little evidence-based effort to optimize such treatments or to identify the characteristics of patients for whom these therapies are most effective and appropriate. A recent questionnaire-based survey of patients treated for SLAP tear indicated that conservative treatment was successful in approximately half (49 percent) of the patients [31]. For these patients, nonoperative therapy, including NSAIDs, posterior capsular stretching and a strengthening program, resulted in significant improvements in pain, function and quality of life. Although the study did not include a comparison to operative treatment, the authors described the functional gains achieved as comparable to those reported in studies of arthroscopic treatment of SLAP tears.

Patients who fail conservative treatment are candidates for arthroscopic therapy. In recent years, the standard surgical approach to glenoid labral tear has been refixation to the glenoid using a suture anchor technique. Of the multiple studies of outcome following this procedure, the majority have focused on repair of superior (SLAP) tears, with emphasis on the more frequent type II tears. In a 2010 systematic review, Gorantla et al. identified 12 studies of repair of type II SLAP lesions with at least two year followup and at least level IV evidence [6]. No study had higher than level III evidence. The percentage of good-to-excellent outcomes ranged from 40 to 94 percent, but was at least 80 percent in seven studies and below 70 percent in only two. In a more recent prospective study (level III), Lenart et al. assessed the outcome of type II SLAP repair in 179 young, active patients drawn from a military population subject to high rates of SLAP injury attributed to traction injury during lifting [7]. At a mean follow-up of 40 months, only 62 percent had outcomes deemed successful.

Reported results for fixation of other regions of the labrum are generally consistent with the findings for SLAP tears. In a study of anterior labral repair in 83 patients with anterior instability, Voos et al. found a failure rate (dislocation or subluxation) of 18 percent [8], consistent with findings in two earlier studies [32,33]. In a level IV study of posterior labral repair in 22 patients with recurrent posterior instability, postoperative recurrence was observed in 2 patients (9 percent) [34].
Overall, results to date indicate that arthroscopic labral repair is a generally effective procedure, but the presence of significant and variable failure rates remains an important concern. Some failures are potentially attributable to the misreading of normal anatomic variants as superior labral tears [35]. In addition, several studies have generated findings suggesting that certain patient populations are at greater risk of failure or incomplete recovery. In five studies of overhead athletes with type II SLAP tears, Gorantla et al. noted that only 64 of 107 athletes (64 percent) returned to their preinjury level of play [6]. Lenart et al. noted the consistency of overhead athlete failure rates with the high failure rate (38 percent) they observed in military patients, who similarly are subject to demanding physical activity [7]. Lenart et al. also found that patients aged $>36$ years were at greater risk of failure (relative risk 3.45). In contrast, Voos et al. observed increased failure of anterior stabilization (38 percent) in patients under 20 years of age [8]. Other risk factors for failure in the Voos et al. study were the presence of large Hill-Sachs lesions and ligamentous laxity. These findings indicate that the decision to proceed with surgery remains a difficult one that requires individualized assessment of risk and expected benefit.

Complications associated with arthroscopic surgery include decreased range of motion, loss of strength, pain, chondrolysis, adhesive capsulitis, nerve injury, osteoarthritis, infection and thromboembolic complications $[9,10]$. While these complications are individually uncommon, the aggregate reported rates of complication range from 4.6 to 10.6 percent [10]. Chondrolysis is a particularly devastating complication that that has proven difficult to treat [36]. A analysis of 375 arthroscopies performed by a community surgeon found a striking association of chondrolysis with the use of lidocaine or bupivacaine anesthetics (49 out of 49 cases) [37]. Associations of chondrolysis with the use of intra-articular thermal energy or contrast media have also been suggested [10]. While these studies point to potential paths for avoidance of postoperative chondrolysis, the complication remains a serious concern.

\section{NEW DIRECTIONS IN TREATMENT OF GLENOID LABRAL TEARS}

The development of arthroscopic procedures has been beneficial to large numbers of patients suffering from shoulder pain. Nevertheless, arthroscopy remains a costly, highly invasive procedure with significant risks of complication. The risk/benefit assessment of the procedure for individual patients is likely to depend on patient age, specific nature of the labral lesion, and goals for postoperative performance level, among other potential factors. These limitations, coupled with the relatively immature stage of research into modalities of nonoperative treatment, suggest that exploration of potential options in conservative care of these disorders may be of considerable benefit to many patients.

Conservative treatment of glenoid labral tears currently focuses on analgesia, rest and rehabilitation. While valuable, these measures do not include any direct effort to support and amplify the spontaneous healing and repair processes in the glenohumeral joint. The central importance of labral healing is underscored by the recent evolution of 
arthroscopic therapy, in which an earlier emphasis on debridement has been replaced by a preference for labral repair by fixation and subsequent healing of the labrum to the glenoid rim. The question then arises as to whether nonsurgical interventions may be found that can similarly support labral healing.

The spontaneous healing process of the glenoid labrum has been recently investigated in an animal model [11]. In this study, the entire inferior half of the rabbit labrum was detached and repositioned to the glenoid without fixation. At one week postoperatively, an inflammatory infiltrate was observed in the dissected clear space. This was followed by the outgrowth of fibrous connective tissue, which subsequently, after three weeks post-operation, acquired dense collagenous fibers. At four weeks post-operation, biomechanical performance had returned to preoperative levels. While the authors note that tissue healing in rabbits is faster than in humans, these observations support the concept of a rapid spontaneous healing process in the labrum. Labral cells have recently been shown to be similar in their gene expression patterns to cells of the meniscus [38], a histologically similar tissue in which proliferative and remodeling responses to inflammatory/wound stimuli are well documented [39]. These considerations support the investigation of interventions that potentially accelerate and enhance labral healing by the introduction of proinflammatory or proliferative stimuli to the labral microenvironment.

\section{REGENERATIVE INJECTION THERAPY (PROLO- THERAPY) FOR MUSCULOSKELETAL REPAIR}

Regenerative injection therapy may be broadly defined as " the injection of growth factors or growth factor production stimulants to promote regeneration of normal cells and tissue.' [40] An example of this approach is the injection of a preparation of enriched platelets (platelet-rich plasma). Activation of the platelets is expected to initiate woundhealing pathways that will support tissue repair. Platelet-rich plasma has been used to treat several musculoskeletal disorders, including osteoarthritis, tendinopathy, bone healing, and muscle injury. Platelet-rich plasma has shown benefit in the treatment of knee osteoarthritis and lateral epicondylitis [41,42]. Positive results have also been obtained, although with less consistency, for a variety of other tendon, bone and muscle pathologies [41,42]. Plateletrich plasma is a simple preparation of a complex material, and as such may be viewed as a potential platform for further development of regenerative strategies. For example, a recent study found that the effects of platelet-rich plasma on collagen production by ligament fibroblasts could be enhanced by the simultaneous presence of autologous peripheral blood mononuclear cells, suggesting a potential direction for modification of therapy [43].

A second strategy for regenerative injection therapy is the administration of irritant or sclerosant substances with the potential to provoke inflammatory and healing responses. The term "prolotherapy" (i.e. proliferative therapy) is frequently used for these treatments, although this term can also be applied to all of regenerative injection therapy, including platelet-rich plasma [40]. The most common injectants for this purpose are hypertonic dextrose $(12-25$ percent) and sodium morrhuate $(0.1-1$ percent $)$. Anesthetics included in the injectant. The mechanistic basis for the action of these agents is not well defined. Hypertonic dextrose, the most frequently used injectant, has been shown to enhance the growth factor responsiveness of multiple cell types, including fibroblasts, vascular smooth muscle cells and ligament cells [44-46]. Preclinical studies with sodium morrhuate have demonstrated the anabolic potential of prolotherapy in tendons and ligaments [47-49].

Several clinical trials have examined the effectiveness of hypertonic dextrose in the treatment of musculoskeletal conditions. Reeves et al. conducted randomized clinical trials (RCTs) of dextrose prolotherapy for osteoarthritis of wrist and knee and showed efficacy for pain reduction and functional improvement [50,51]. In an RCT of prolotherapy (dextrose + sodium morrhuate) for lateral epicondylitis, Scarpone et al. showed significant gains in pain score and grip strength that persisted for at least one year [52]. An RCT carried out by Yelland et al. compared dextrose prolotherapy, eccentric loading exercise, or combined therapy for Achilles tendinosis. Prolotherapy as monotherapy, and particularly in combined treatment, provided more rapid reductions of pain, stiffness, and functional limitations [53]. The evidence for efficacy of prolotherapy in low back pain is less consistent: a Cochrane review found five high-quality RCTs [54], two of which had positive findings for prolotherapy in combination with other treatments.

\section{HYPERTONIC DEXTROSE TREATMENT OF GLENOID LABRAL TEAR: A RETROSPECTIVE CASE SERIES}

In view of the evidence that regenerative injection therapy can stimulate connective tissue growth responses and provide clinical benefit with low morbidity in musculoskeletal settings, including joint disorders, we were encouraged to explore this therapeutic approach as a potential means of enhancing conservative treatment and providing an alternative to surgery for patients with shoulder pain and labral tear. Here we describe our experience with this therapeutic approach in a consecutive series of 33 patients diagnosed with glenoid labral tear.

\section{Patients}

This study concerns 33 patients (21 men and 12 women) who presented at our chronic pain clinic with shoulder pain. The average age of the patients was $32.4(15-62)$ years. Patients reported having pain for a mean of 19 months prior to visit. At physical exam, all patients had crunching and grinding with shoulder motion. All patients had at least three positive results among the following tests: crank test, O'Brien test, Speed's test, and tenderness of the bicipital groove. The diagnosis of labral tear indicated by these findings was confirmed in 15 patients by MRI. Three patients had previous surgery for labral tear and continued to complain of pain.

\section{Intervention}

All patients were treated with dextrose prolotherapy, for which the injectant contained 15\% Dextrose, $0.1 \%$ Procaine, and $10 \%$ Sarapin. The area to be treated was anesthetized with 5\% lidocaine cream and cleaned with a $3 \%$ hydrogen 
peroxide, followed by a $2 \%$ chlorhexadine gluconate solution. A total of $48 \mathrm{cc}$ at 30 locations, including the posterior joint capsule, the supraspinatus, infraspinatus and teres minor tendons, and the glenohumeral, coracohumeral and acromioclavicular ligaments. The glenohumeral joint received $8 \mathrm{cc}$. For 14 patients, the injectant also contained $2.5-5 \mathrm{mg} / \mathrm{cc}$ sodium morrhuate. This treatment was repeated an average of 3.4 times with $3-6$ week intervals between sessions. Nine patients received additional treatment with platelet-rich plasma. For this treatment, $60 \mathrm{cc}$ autologous blood, anticoagulated with Anticoagulant Citrate Dextrose Solution Formula A, USP (ACD-A), was separated in a platelet-rich plasma centrifuge for 15 minutes. Plateletpoor plasma was drawn off and $11 \mathrm{cc}$ platelet-rich plasma collected for injection into and around shoulder joints (7 cc intra-articular) that had been anesthetized with $1 \mathrm{cc}$ of $8.0 \%$ procaine $(80 \mathrm{mg})$.

\section{Clinical Outcomes}

At final interview, a questionnaire was administered in which patients used a numeric rating scale (NRS) to assess pre-treatment and post-treatment pain intensity (at rest, during normal activity and during exercise), stiffness, range of motion and crunching. Multiple choice questions were used to assess pre- and post-treatment ability to exercise. The choices included: exercise totally compromised (unable to exercise); severe compromise (able to exercise 0-30 minutes); moderate compromise (able to exercise 30-60 minutes); mild compromise (able to exercise $>60$ minutes, but not as much as desired); no compromise (can exercise without limit). Patients were also asked how many pills and how many pain medications they took for their condition pre- and post-treatment.

\section{Statistical Analysis}

The distributions for baseline values were non-normal by Shapiro-Wilk test. Post-treatment and pre-treatment values were compared by Wilcoxon signed rank test. Differences with $p$ values $\leq 0.01$ were considered significant.

\section{RESULTS}

Patient-reported outcomes were obtained at a mean of 16 months after completion of treatment (range $1-42$ months). We observed highly significant reductions in pain at rest, pain during normal activity, and pain during exercise (Fig. 1). For pain at rest, NRS scores declined from 5.1 \pm 3.5 pretreatment to $0.3 \pm 0.7$ post-treatment $(\mathrm{p}<0.0001)$. For pain during normal activity, scores declined from $6.0 \pm 2.9$ to $0.2 \pm 0.7 \quad(\mathrm{p}<0.0001)$, and for pain during exercise from $7.6 \pm 2.9$ to $0.6 \pm 1.4(\mathrm{p}<0.0001)$. Pain ratings in these three categories were reduced to $5 \%, 4 \%$ and $7 \%$ of their baseline values, respectively. All patients who reported pain before treatment had reduced pain after treatment. Pain reduction did not show any dependence on the interval between treatment completion and patient report (Fig. 2), suggesting that gains were maintained for up to 40 months following the final treatment session. For stiffness, range of motion, crunching, medications and pill consumption, only patients with non-zero baseline values were analyzed. 30 patients had non-zero baseline for at least one of these categories, and 27 had non-zero baseline for at least two categories. Overall, patients reported gains in $93 \%$ (84 of 90) of the instances in which they reported non-zero baseline values for these categories. For stiffness, range of motion and crunching, post-treatment ratings were reduced to $5 \%, 4 \%$ and $33 \%$ of baseline values, respectively (Fig. 1). Notably, daily pill consumption for alleviation of symptoms, the most objective of our patient-reported outcomes, was nearly eliminated: only one of the 15 patients who reported pill use before treatment reported any pill use post-treatment. Only one patient reported adverse results: an increase in stiffness and reduced range of motion.

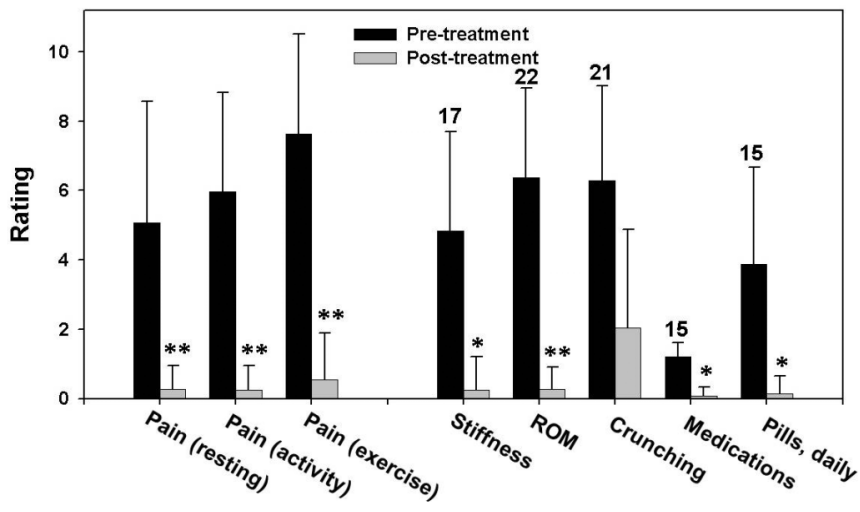

Fig. (1). Patient-reported quantitative outcomes. Patients reported pain, stiffness, range of motion (ROM) and crunching on a $0-10$ rating scale. For consumption of medications, the ordinate represents the number of medications used or the number of daily pills taken. For pain reports, $n=33$. For stiffness, ROM, crunching and medications, only reports with non-zero baseline values were analyzed, the numbers of which are indicated above the bars. $* p \leq 0.01, * * p \leq 0.0001$ by Wilcoxon signed-rank test.

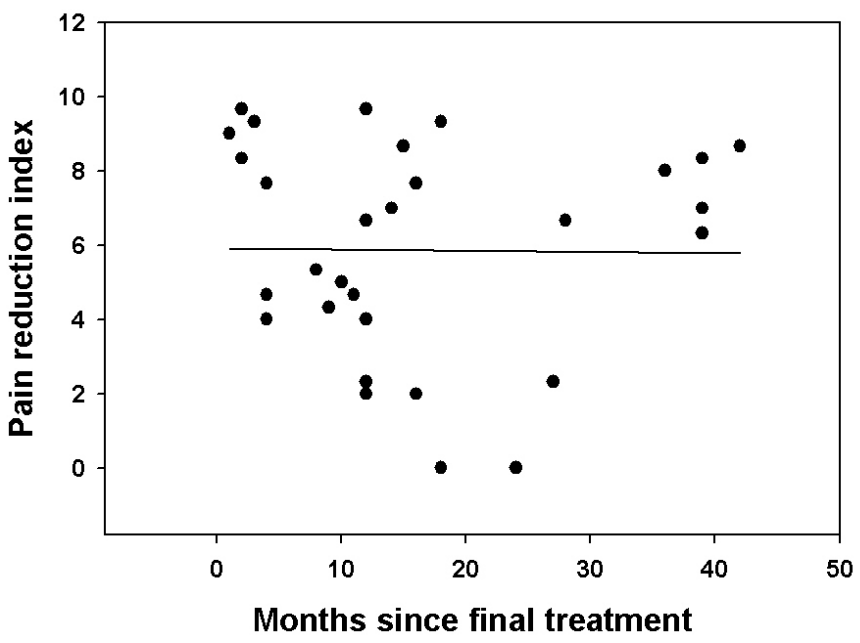

Fig. (2). Time-dependence of pain relief after treatment. A pain reduction index was calculated for each patient by subtracting the post-treatment from the pre-treatment value for each of the three pain categories, and then obtaining the mean of the three differences. The line on the scatter plot represents a least-squares linear regression.

Patients reported gains in functionality with respect to the ability to exercise (Fig. 3). All 31 patients who reported exercise limitation at baseline experienced improvement in this area. Twenty-nine of these patients reported an ability to return to unlimited exercise after treatment, and none reported either total or severely compromised exercise 
capability. No adverse outcomes were reported with respect to exercise.

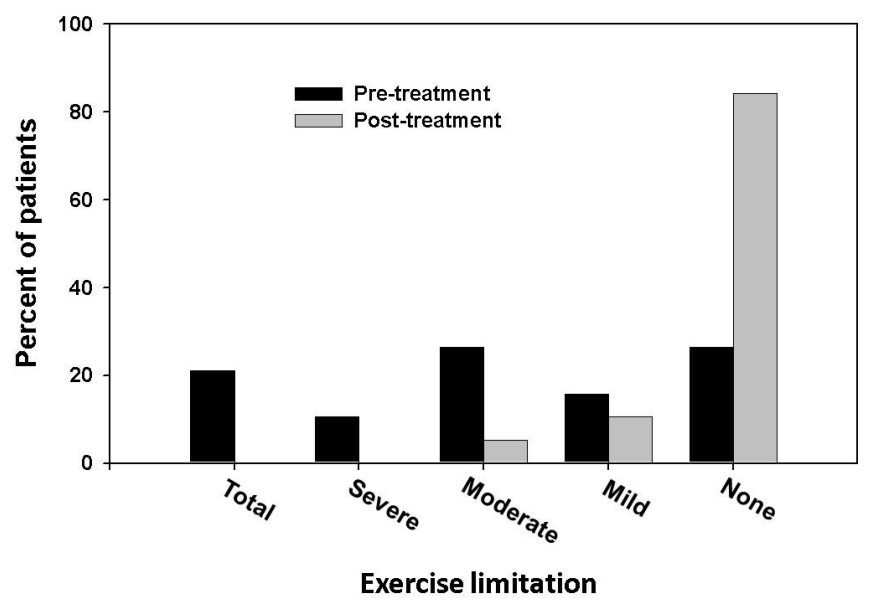

Fig. (3). Patient-reported functionality. Patients chose from five possibilities to describe the degree of compromise in their ability to exercise. $\mathrm{n}=33$.

Overall, the 33 patients in the study reported a total of 207 symptoms prior to treatment (three categories of pain, stiffness, range of motion, crunching, exercise limitation). Following treatment, patients reported complete relief (best possible score) for 142 out of these 207 complaints (69\%).

Thirty-two of the 33 patients in the study expressed satisfaction with treatment. The exception was a patient who experienced gains after each session that were not maintained. Thirteen patients reported that surgery had been recommended by a previous physician, and all 13 reported that they no longer believed that they needed surgery.

\section{DISCUSSION}

The case series described here represents the first study of regenerative injection therapy for glenoid labral lesions. The results of the study were encouraging, as all patients with pain reported pain reduction and all patients with exercise impairment reported improved ability to exercise, in most cases resulting in a return to pre-injury levels. All but one patient expressed a positive view of their treatment on the questionnaire. Although our average follow up was 16 months after the last prolotherapy session, improvements appeared to be stable during at least the first three years posttreatment, as judged by the lack of time dependence for pain reduction.

This pilot study has a number of limitations. Without a control group, we cannot conclude that therapy was effective. It is possible that some patient gains are the result of spontaneous improvement. However, the mean duration of pain prior to treatment was 19 months, and we did not detect any time dependence in symptomatic improvement, which for seven patients was already observed within the first four months post-treatment. These findings suggest that the reported gains are largely the result of treatment. Additional limitations include the reliance on post-hoc questionnaires and the paucity of objective outcome measures. The nearly complete absence of pain medication use after treatment provides a degree of objective confirmation of patient benefit. With respect to post-hoc reporting, the expected confounding effect is the exaggeration of gain by inflation of baseline values. However, our data are notable for the high frequency of post-treatment reports of complete symptomatic relief, rather than partial relief from high baseline values, suggesting that post-hoc reporting is not a major confounding factor in the study. A further limitation is the use of individualized treatments, which included supplementation with plateletrich plasma for nine patients and addition of sodium morrhuate for 14 patients. These variations, however, cannot account for the consistency of symptomatic improvement observed throughout the patient set.

Hypertonic dextrose has multiple effects that may potentially support labral healing, including the stimulation of growth factor production and proliferative responses [44-46], as well as the possible provocation of inflammatory processes [55] that may promote angiogenic and healing responses. The nature of healing responses in the labrum is still poorly understood, and regenerative injection therapy has the merit of offering broadly acting stimuli that can potentially enhance and amplify the healing process. Given the limited and uncertain efficacy of current conservative treatment of glenoid labral tear, and the risks, failure rates and expense associated with arthroscopy, regenerative therapy may be viewed as a potential adjunct to conservative management that deserves investigation, both in animal models of labral tear and in expanded and controlled clinical studies.

\section{CONCLUSIONS}

Uncertainties in the management of glenoid labral lesions, in particular with respect to the efficacy of conservative treatments, provide a rationale for the investigation of regenerative injection therapy for labral tear. Our initial experience with hypertonic dextrose in patients with glenoid labral tear suggests that the procedure is safe and potentially efficacious. We observed substantial gains in pain relief and functionality in a large majority of patients.

\section{CONFLICT OF INTEREST}

The authors confirm that this article content has no conflict of interest.

\section{ACKNOWLEDGEMENTS}

Declared none.

\section{REFERENCES}

[1] Stevenson JH, Trojian T. Evaluation of shoulder pain. J Fam Pract 2002; 51: 605-11.

[2] Cherry DK, Hing E, Woodwell DA, Rechtsteiner EA. National Ambulatory Medical Care Survey: 2006 summary. Natl Health Stat Report 2008: 1-39.

[3] Cadogan A, Laslett M, Hing WA, Mcnair PJ, Coates MH. A prospective study of shoulder pain in primary care: Prevalence of imaged pathology and response to guided diagnostic blocks. BMC Musculoskeletal Disorders 2011; 12: 119.

[4] Kim TK, Queale WS, Cosgarea AJ, McFarland EG. Clinical features of the different types of SLAP lesions - An analysis of one hundred and thirty-nine cases. J Bone and Joint Surg-Am 2003; 85A: 66-71.

[5] D'Alessandro DF, Fleischli JE, Connor PM. Superior labral lesions: Diagnosis and management. J Athletic Train 2000; 35: 286-92.

[6] Gorantla K, Gill C, Wright RW. The Outcome of Type II SLAP Repair: A Systematic Review. Arthroscopy-the J Arthroscop Related Surg 2010; 26: 537-45. 
[7] Provencher MT, McCormick F, Dewing C, McIntire S, Solomon D. A prospective analysis of 179 type 2 superior labrum anterior and posterior repairs: outcomes and factors associated with success and failure. Am J Sports Med 2013; 41(4): 880-886.

[8] Voos JE, Livermore RW, Feeley BT, et al. Prospective evaluation of arthroscopic bankart repairs for anterior instability. Am J Sports Med 2010; 38: 302-7.

[9] Marecek GS, Saltzman MD. Complications in shoulder arthroscopy. Orthopedics 2010; 33: 492-7.

[10] Kang RW, Frank RM, Nho SJ, et al. Complications associated with anterior shoulder instability repair. Arthroscopy-the J Arthroscop Related Surg 2009; 25: 909-20.

[11] Abe H, Itoi E, Yamamoto N, et al. Healing processes of the glenoid labral lesion in a rabbit model of shoulder dislocation. Tohoku J Exp Med 2012; 228: 103-8.

[12] Williams MM, Snyder SJ, Buford D. The buford complex - the cord-like middle glenohumeral ligament and absent anterosuperior labrum complex - a normal anatomic capsulolabral variant. Arthroscopy 1994; 10: 241-7.

[13] Rao AG, Kim TK, Chronopoulos E, McFarland EG. Anatomical variants in the anterosuperior aspect of the glenoid labrum - A statistical analysis of seventy-three cases. J Bone Joint Surg-Am 2003; 85A: 653-9.

[14] Bedi A, Allen AA. Superior labral lesions anterior to posteriorevaluation and arthroscopic management. Clin Sports Med 2008; 27: 607 .

[15] Vangsness CT, Jorgenson SS, Watson T, Johnson DL. The origin of the long head of the biceps from the scapula and glenoid labrum - an anatomical study of 100 shoulders. J Bone Joint Surg-Br 1994; 76B: 951-4.

[16] Tischer T, Putz R. Anatomy of the superior labrum complex of the shoulder. Orthopade 2003; 32: 572.

[17] Hertz H, Weinstabl R, Grundschober F, Orthner E. Macroscopic and microscopic anatomy of the shoulder joint and the limbus glenoidalis. Acta Anat (Basel) 1986; 125: 96-100.

[18] Fehringer EV, Schmidt GR, Boorman RS, Churchill RS, Smith KL, Norman AG, Sidles JA, Matsen FA. The anteroinferior labrum helps center the humeral head on the glenoid. J Shoulder Elbow Surg 2003; 12: 53-8.

[19] Habermeyer P, Schuller U, Wiedemann E. The intra-articular pressure of the shoulder: an experimental study on the role of the glenoid labrum in stabilizing the joint. Arthroscopy 1992; 8: 16672.

[20] Hill AM, Hoerning EJ, Brook K, et al. Collagenous microstructure of the glenoid labrum and biceps anchor. J Anat 2008; 212: 853-62.

[21] Cooper DE, Arnoczky SP, Obrien SJ, Warren RF, Dicarlo E, Allen AA. Anatomy, histology, and vascularity of the glenoid labrum. J Bone Joint Surg-Am 1992; 74A: 46-52.

[22] Prodromos CC, Ferry JA, Schiller AL, Zarins B. Histological studies of the glenoid labrum from fetal life to old-age. J Bone and Joint Surg-Am 1990; 72A: 1344-8.

[23] Taylor M, Fruth S, Kegerreis S. The glenoid labrum: basic science and clinical correlation. J Sport Rehab 1995; 4: 42-53.

[24] Rames RD, Karzel RP. Injuries to the Glenoid Labrum, Including Slap Lesions. Orthop Clin North Am 1993; 24: 45-53.

[25] Bankart AS, Cantab MC. Recurrent or habitual dislocation of the shoulder-joint. Clin Orthop Relat Res 1993:3-6.

[26] Snyder SJ, Banas MP, Karzel RP. An analysis of 140 injuries to the superior glenoid labrum. J Shoulder Elbow Surg 1995; 4: 243-8.

[27] Handelberg F, Willems S, Shahabpour M, Huskin JP, Kuta J: SLAP lesions. A retrospective multicenter study. Arthroscopy 1998; 14: 856-62.

[28] Jee WH, McCauley TR, Katz LD, Matheny JM, Ruwe PA, Daigneault JP. Superior labral anterior posterior (SLAP) lesions of the glenoid labrum: Reliability and accuracy of MR arthrography for diagnosis. Radiology 2001; 218: 127-32.

[29] Walsworth MK, Doukas WC, Murphy KP, Mielcarek BJ, Michener LA: Reliability and diagnostic accuracy of history and physical examination for diagnosing glenoid labral tears. Am J Sports Med 2008; 36: 162-8.

[30] Smith TO, Drew BT, Toms AP. A meta-analysis of the diagnostic test accuracy of MRA and MRI for the detection of glenoid labral injury. Arch Orthop Trauma Surg 2012; 132: 905-19.

[31] Edwards SL, Lee JA, Bell JE, et al. Nonoperative treatment of superior labrum anterior posterior tears improvements in pain, function, and quality of life. Am J Sports Med 2010; 38: 1456-61.
[32] Cho NS, Hwang JC, Rhee YG.rthroscopic stabilization in anterior shoulder instability: Collision athletes versus noncollision athletes. Arthroscopy- J Arthroscop Relat Surg 2006; 22: 947-53.

[33] Jones KJ, Wiesel B, Ganley TJ, Wells L. Functional outcomes of early arthroscopic Bankart repair in adolescents aged 11 to 18 years. J Pedia Orthopaed 2007; 27: 209-13.

[34] Lenart BA, Sherman SL, Mall NA, Gochanour E, Twigg SL, Nicholson GP. Arthroscopic Repair for Posterior Shoulder Instability. Arthroscopy- J Arthroscop Relat Surg 2012; 28: 133743.

[35] Burns JP, Bahk M, Snyder SJ. Superior labral tears: repair versus biceps tenodesis. J Shoulder Elbow Surg 2011; 20: S2-S8.

[36] Scheffel PT, Clinton J, Lynch JR, Warme WJ, Bertelsen AL, Matsen FA. Glenohumeral chondrolysis: A systematic review of 100 cases from the English language literature. J Shoulder Elbow Surg 2010; 19: 944-9.

[37] Wiater BP, Neradilek MB, Polissar NL, Matsen FA. Risk Factors for Chondrolysis of the Glenohumeral Joint A Study of Three Hundred and Seventy-five Shoulder Arthroscopic Procedures in the Practice of an Individual Community Surgeon. J Bone Joint SurgAm 2011; 93A: 615-23.

[38] Dhollander AAM, Lambrecht S, Verdonk PCM, et al. First insights into human acetabular labrum cell metabolism. Osteoarthritis Cartil 2012; 20: 670-7.

[39] Tan GK, Cooper-White JJ. Interactions of meniscal cells with extracellular matrix molecules towards the generation of tissue engineered menisci. Cell Adhesion Migration 2011; 5: 220-6.

[40] Reeves KD, Fullerton BD, Topol G. Evidence-based regenerative injection therapy (prolotherapy) in sports medicine. In The Sports Medicine Resource Manual. In: Seidenberg PH, Beutler PI. Eds. Elsevier 2008: pp. 611-9.

[41] Taylor DW, Petrera M, Hendry M, Theodoropoulos JS. A systematic review of the use of platelet-rich plasma in sports medicine as a new treatment for tendon and ligament injuries. Clin J Sport Med 2011; 21: 344-52.

[42] Steinert AF, Middleton KK, Araujo PH, Fu FH. Platelet-rich plasma in orthopaedic surgery and sports medicine: pearls, pitfalls and new trends in research . Oper Tech Orthop 2012; 22: 91-103.

[43] Yoshida R, Murray MM. Peripheral blood mononuclear cells enhance the anabolic effects of platelet-rich plasma on anterior cruciate ligament fibroblasts. J Orthop Res 2012; 31(3): 29-34.

[44] Fukuda K, Kawata S, Inui Y, et al. High concentration of glucose increases mitogenic responsiveness to heparin-binding epidermal growth factor-like growth factor in rat vascular smooth muscle cells. Arteriosclerosis Thrombo Vasc Bio 1997; 17: 1962-8.

[45] Ohgi S, Johnson PW. Glucose modulates growth of gingival fibroblasts and periodontal ligament cells: Correlation with expression of basic fibroblast growth factor. J Periodontal Res 1996; 31: 579-88.

[46] Pugliese G, Pricci F, Locuratolo N, et al. Increased activity of the insulin-like growth factor system in mesangial cells cultured in high glucose conditions. Relation to glucose-enhanced extracellular matrix production. Diabetologia 1996; 39: 775-84.

[47] Liu YK, Tipton CM, Matthes RD, Bedford TG, Maynard JA, Walmer HC. An in situ study of the influence of a sclerosing solution in rabbit medial collateral ligaments and its junction strength. Connect Tissue Res 1983; 11: 95-102.

[48] Maynard JA, Pedrini VA, Pedrinimille A, Romanus B, Ohlerking F. Morphological and biochemical effects of sodium morrhuate on tendons. J Orthop Res 1985; 3: 236-48.

[49] Aneja A, Karas SG, Weinhold PS, Afshari HM, Dahners LE. Suture plication, thermal shrinkage, and sclerosing agents - Effects on rat patellar tendon length and biomechanical strength. Am J Sports Med 2005; 33: 1729-34.

[50] Reeves KD, Hassanein K. Randomized prospective double-blind placebo-controlled study of dextrose prolotherapy for knee osteoarthritis with or without ACL laxity. Altern Ther Health Med 2000; 6: 68 .

[51] Reeves KD, Hassanein K. Randomized, prospective, placebocontrolled double-blind study of dextrose prolotherapy for osteoarthritic thumb and finger (DIP, PIP, and trapeziometacarpal) joints: Evidence of clinical efficacy. J Altern Complement Med 2000; 6: 311-20.

[52] Scarpone M, Rabago DP, Zgierska A, Arbogast G, Snell E. The efficacy of prolotherapy for lateral epicondylosis: A pilot study. Clin J Sport Med 2008; 18: 248-54. 
[53] Yelland MJ, Sweeting KR, Lyftogt JA, Shu KN, Scuffham PA, Evans KA. Prolotherapy injections and eccentric loading exercises for painful Achilles tendinosis: a randomised trial. Br J Sports Med 2011; 45: 421-8.
[54] Dagenais S, Yelland MJ, Del Mar C, Schoene ML. Prolotherapy injections for chronic low-back pain. Cochrane Database of Syst Rev 2007.

[55] Jensen KT, Rabago DP, Best TM, Patterson JJ, Vanderby R. Early inflammatory response of knee ligaments to prolotherapy in a rat model. J Orthop Res 2008; 26: 816-23.

(C) Hauser et al.; Licensee Bentham Open.

This is an open access article licensed under the terms of the Creative Commons Attribution Non-Commercial License (http: //creativecommons.org/licenses/by$\mathrm{nc} / 3.0 /$ ) which permits unrestricted, non-commercial use, distribution and reproduction in any medium, provided the work is properly cited. 